\title{
A Catalog of Bipolar Active Regions Violating the Hale Polarity Law, 1989-2018
}

\author{
Anastasiya Zhukova ${ }^{1}$ [D $\cdot$ Anna Khlystova ${ }^{2}$ [D] \\ Valentina Abramenko ${ }^{1}$ (ID) \\ Dmitry Sokoloff $3,4,5$
}

(C) Springer $\bullet \bullet \bullet \bullet$

\begin{abstract}
There is no list of bipolar active regions (ARs) with reverse polarity (anti-Hale regions), although statistical investigations of such ARs (bearing the imprint of deep subphotospheric processes) are important for understanding solar-cycle mechanisms. We studied 8606 ARs from 1 January 1989 to 31 December 2018 to detect anti-Hale regions and to compile a catalog. The Solar and Heliospheric Observatory (SOHO) and the Solar Dynamics Observatory (SDO) data, as well as the Debrecen Photoheliographic Data, the Mount Wilson Observatory catalog and drawings, and the USAF/NOAA Solar Region Summary were used. Complex, ambiguous cases related to anti-Hale region identification were analyzed. Two basic and four additional criteria to identify an AR as an anti-Hale region were formulated. The basic criteria assume that: i) dominating features of an AR have to form a bipole of reverse polarity with sunspots/pores
\end{abstract}

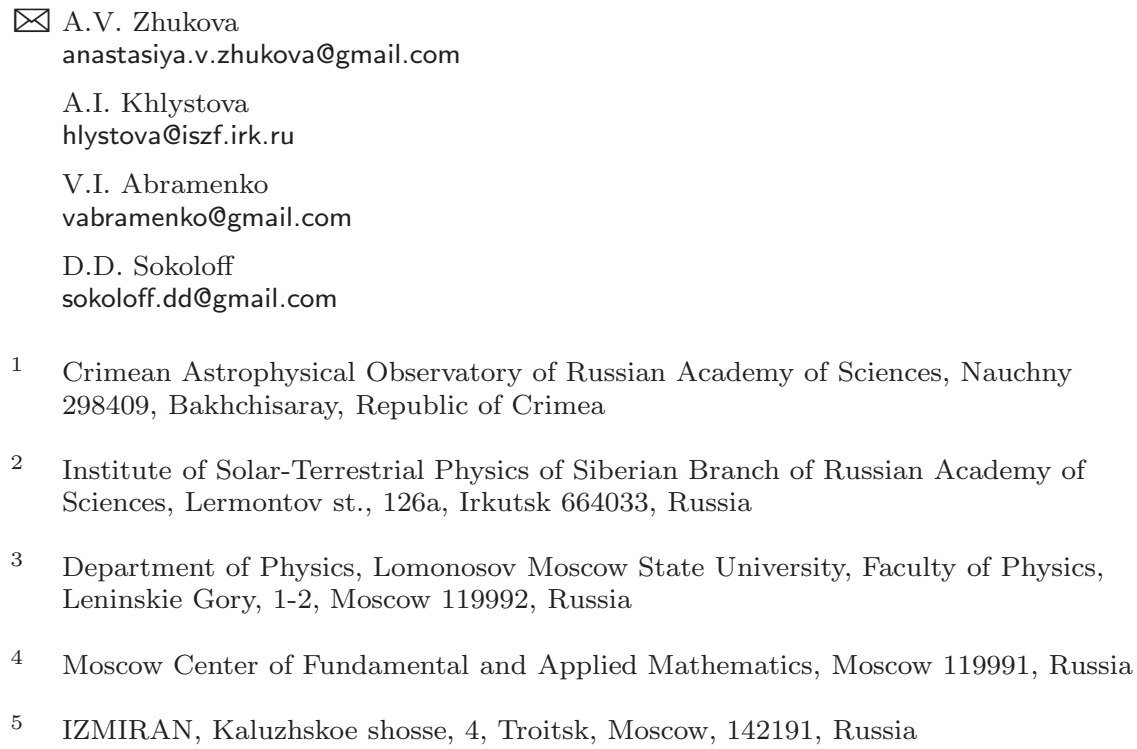



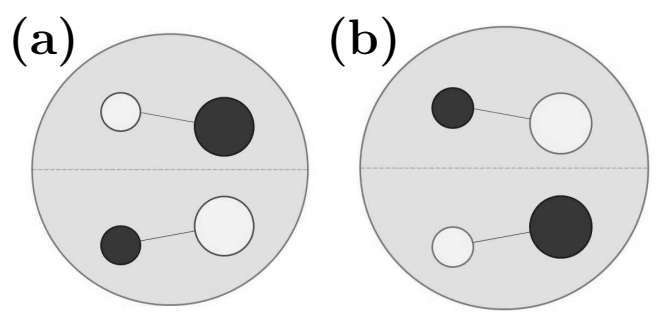

Figure 1. Regular (with the leading sunspot obeying Hale's polarity law) bipolar ARs in even (a) and odd (b) cycles. Sunspots with negative (positive) polarity are shown as black (white). The polarity of sunspots of anti-Hale regions is opposite.

of both polarities being present; ii) magnetic connections between the opposite polarities has to be observed. A catalog of anti-Hale regions (275 ARs) is compiled. The catalog contains: NOAA number, date of the greatest total area of sunspots, coordinates, and corrected sunspot area for this date. The tilt and the most complex achieved Mount Wilson magnetic class are also provided. The percentage of anti-Hale groups meeting the proposed criteria is $\approx 3.0 \%$ from all studied ARs, which is close to early estimations by authors who had examined each AR individually: $\approx 2.4 \%$ by Hale and Nicholson (Astrophys. J. 62, 270, 1925) and $\approx 3.1 \%$ by Richardson (Astrophys. J. 107, 78, 1948). The enchancement of the anti-Hale percentage in later research might be related to: i) increasing sensitivity of instruments (considering smaller and smaller bipoles); ii) the ambiguities in the anti-Hale region identification. The catalog is available as the Electronic Supplementary Material and at the CrAO website (sun.crao.ru/databases/catalog-anti-hale/).

Keywords: Active Regions, Structure; Solar Cycle, Observations; Sunspots, Statistics

\section{Introduction}

The majority of active regions (ARs) obey the empirical rules for sunspot groups (Hale et al., 1919): Hale's polarity law, Joy's law, etc. (regular ARs). The pioneering models of the magnetic cycle (Parker, 1955; Babcock, 1961; Leighton, 1964), as well as the global mean-field dynamo theory (see, e.g., Moffatt, 1978; Krause and Rädler, 1980), explain well the existance of the regular ARs. However, ARs violating these empirical rules also occur. According to Hale's law during an even (odd) sunspot cycle, the leading sunspot of a bipolar AR has negative (positive) polarity in the northern hemisphere and the situation is reversed in the southern hemisphere. A sketch of regular ARs of even (odd) cycles is presented as Figure 1. In this article, we deal with bipole sunspot groups with the reverse polarity (anti-Hale regions). We do not consider here other types of irregularities.

Anti-Hale ARs bear the imprint of deep subphotospheric processes. So, statistical studies of these ARs may shed light on our understanding of solar magneticfield evolution and solar-cycle mechanisms. For instance, Sokoloff, Khlystova, and Abramenko (2015) found that a contribution of small-scale fluctuation dynamo in the interplay with the global dynamo is more pronounced at solar-cycle 
minimum. The authors came to this conclusion based on their probabilistic model and the revealed enhancement of the relative number of anti-Hale regions during solar minimum. Note that the presence of magnetic-field fluctuations on smaller (as compared to the mean-field) scales was earlier predicted by both the turbulent dynamo theory (e.g. Kraichnan and Nagarajan, 1967; Kazantsev, 1968; Zeldovich, Ruzmaikin, and Sokoloff, 1990; Brandenburg and Subramanian, 2005) and direct numerical simulations (e.g. Blackman and Field, 2002; Rädler, Kleeorin, and Rogachevskii, 2003; Sur, Brandenburg, and Subramanian, 2008; Brandenburg, Sokoloff, and Subramanian, 2012). The decay of anti-Hale ARs can explain the weakening of the polar magnetic field: the transport of remnants of the following parts of anti-Hale regions to the poles causes the decrease of the polar field (Mordvinov and Kitchatinov, 2019). Numerical simulations by Hazra, Choudhuri, and Miesch (2017) show that the high-latitude anti-Hale ARs in the middle phase of a cycle must contribute to weakening of the polar field more than the rest of anti-Hale groups. Jiang, Cameron, and Schüssler (2015) also found that the magnetic-flux advection through the Equator due to decay of anti-Hale ARs causes the weakening of the process of the polar-field recovery. Determination of the magnetic twist and writhe for several ARs attributed as anti-Hale regions allowed López Fuentes et al. (2000, 2003) to estimate the degree of influence of various mechanisms (kink-instability, effect of the Coriolis force, etc.) on the deformation of flux-tubes. Thus, the observational data on the statistics and properties of anti-Hale groups is in high demand in the solar physics community.

Unfortunately, there is no reliable database of anti-Hale regions. Although many catalogs provide information on the polarity of leading and following parts of ARs and use this information (for example, to measure the tilt of the sunspotgroup axis), the catalogs do not provide these data explicitly. The Mount Wilson Observatory (MWO) catalog had been a main data source on the anti-Hale ARs for a long time. The catalog had been replenished irregularly since 1920. The data are available digitally since 1962 . From 1989 to 2004 the ARs of reverse polarity are marked with the sign "+". According to many researchers who examined the catalog, some ARs in the catalog are marked with this sign erroneously. Thus, Hale and Nicholson (1925) viewed a lot of AR drawings to verify the magnetic class detected by MWO operators. Richardson (1948) reported checking each AR of reverse polarity from the MWO catalog based on the initial records. Errors in anti-Hale region identification in the MWO catalog were also revealed by Sokoloff, Khlystova, and Abramenko (2015).

The published estimation of the relative number of anti-Hale regions varies within a rather wide range: from a few percent to approximately $(8-9) \%$. Hale and Nicholson (1925) found that $<6$ (2.4 on average) of all ARs are the reversepolarity groups with simple bipolar magnetic configuration. Later the following results for this parameter were reported: 3.1 (Richardson, 1948), <5 (Smith and Howard, 1968), 1.8 (Vitinsky, 1986), 4 (Wang and Sheeley, 1989); 4.9 (Khlystova and Sokoloff, 2009, Sokoloff and Khlystova, 2010), 4 (Stenflo and Kosovichev, 2012), $\approx 8$ (Li and Ulrich, 2012; McClintock, Norton, and Li, 2014; Li, 2018). Note that only part of these studies were performed on the basis of the MWO catalog. Many authors used their own databases. McClintock, Norton, and Li 
(2014) suggested that the inhomogeneity of the data used might be the reason for such a discrepancy in the results of various authors. The size of included ARs is one of the main reasons for discrepancy, because small sunspot groups are prone to violate the Hale's polarity law more frequently (Richardson, 1948; Howard, 1989; Stenflo and Kosovichev, 2012). Only $\approx 60 \%$ of ephemeral regions obey this law (Hagenaar, 2001). Moreover, the smallest quiet-Sun bipoles are randomly oriented (Tlatov, Vasil'eva, and Pevtsov, 2010).

Errors in the identification of anti-Hale regions also occure. For example, there is an obvious difference in numbers and coordinates of anti-Hale ARs on the time-latitude diagrams reported by Richardson (1948) and by Vitinsky (1986) in spite of the fact that the authors used the same database. The known reasons for anti-Hale region misidentification are: i) erroneous detection of ARs boundaries (Sokoloff, Khlystova, and Abramenko, 2015); ii) the wrong distribution of ARs between cycles during the transition from one solar cycle to anoter; iii) the misalignment between the magnetic and the heliographic Equators. The two latter reasons are discussed by McClintock, Norton, and Li (2014).

When compiling a database for further identification of anti-Hale regions, the type of the source data (magnetograms, drawings, etc.) is also important. Thus, Stenflo and Kosovichev (2012) reported about the objective difficulties in automatic detection of individual sunspot groups from full-disk magnetograms and extraction of single ARs. Besides, inaccuracies related to detection of weighted centers of polarities may also produce errors. Wang et al. (2015) revealed that white-light measurements generally underestimate the tilts (axial inclinations) of ARs, even when the leading- and the following-polarity sectors are identified correctly.

To summarize, studies of anti-Hale regions are essential for understanding the solar dynamo. So far, there is no reliable list of ARs with reverse polarity. We undertake a compilation of such a database starting from 1989 and covering the last two and a half solar cycles. We paid special attention to the analysis of mistakes and ambiguities in identification of anti-Hale ARs.

\section{Data and Method}

\subsection{Data Sources}

To examine ARs visually, we used the full-solar-disk daily data: magnetograms, continuum and extreme ultraviolet (EUV) images, sketches and drawings, as well as different images of the individual ARs. We also used available catalogs containing the information on the leading sunspot polarity of bipolar ARs and other necessary data. We used the following sources:

- the Michelson Doppler Imager (MDI: Scherrer et al., 1995) and the Extreme Ultraviolet Imaging Telescope (EIT: Moses et al., 1997) on board the Solar and Heliospheric Observatory (SOHO);

- the Helioseismic and Magnetic Imager (HMI: Scherrer et al., 2012) and the Atmospheric Imaging Assembly (AIA: Lemen et al., 2012) onboard the Solar Dynamic Observatory (SDO); 
- the Debrecen Photoheliographic Data (DPD)

(Baranyi, Győri, and Ludmány, 2016) accessible from

fenyi.solarobs.csfk.mta.hu/DPD/;

- the websites www.helioviewer.org (Helioviewer), www.solarmonitor.org (Solar Monitor);

- the MWO drawings - which are available in the digital archive at $\mathrm{ftp}$ //howard.astro.ucla.edu/pub/obs/drawings/;

- the MWO catalog accessible at the National Geophysical Data Center at ftp://ftp.ngdc.noaa.gov/STP/SOLAR_DATA/SUNSPOT_REGIONS/

Mt_Wilson/;

- the Crimean Astrophysical Observatory (CrAO) catalog

(Abramenko, Zhukova, and Kutsenko, 2018; Zhukova, 2018) at

sun.crao.ru/databases/catalog-mmc-ars;

- the United States Air Force/National Oceanic and Atmospheric Administration Solar Region Summary (USAF/NOAA SRS) at

solarcyclescience.com/activeregions.html.

Note that at the biginning of our study we also used the Royal Observatory, Greenwich - USAF/NOAA Sunspot Data provided by the National Aeronautics and Space Administration (NASA). But these data are available only until October 2016 (solarscience.msfc.nasa.gov/greenwch.shtml), and later we restricted ourselves to the USAF/NOAA SRS database.

\subsection{Ambiguities in Anti-Hale Region Identification}

We encountered the following ambiguities in anti-Hale AR identification.

i) During solar-cycle minima the high-latitude sunspot groups (new-cycle precursors) might be erroneously accepted as reverse-polarity ARs. The periods of this ambiguity are 1996 and 2008 (the cycle minima). To determine what cycle a suspicious high-latitude group belongs to, we used a technique suggested by McClintock, Norton, and Li (2014). The boundaries between cycles in North and South hemispheres were defined as:

$$
60 \times \text { ylat }= \pm(\text { xdate }-x i n t),
$$

where ylat is latitude, xdate is a function of a number of days from 1 January 1974, and xint (a point of inter section at the Equator) was visually determined separately for the best fit for each cycle. The time-latitude diagram based on the USAF/NOAA SRS sunspot-group data is presented as Figure 2 Cycle boundaries for Solar Cycles 22/23 and 23/24 are plotted from McClintock, Norton, and $\mathrm{Li}(2014)$ data. We also plotted here the fit of the boundary between Solar Cycles 24 and 25. This boundary might be slightly changed with Solar Cycle 25 evolution.

ii) Detection of individual sunspot groups in large composite activity complexes represents a challenge. Stenflo and Kosovichev (2012) supposed that it does not seem feasible to make a program that can automatically and reliably identify all of the bipolar regions. Blocks and chains of ARs are typical for solar maxima. In that and some other cases sunspots/pores may be attributed with 


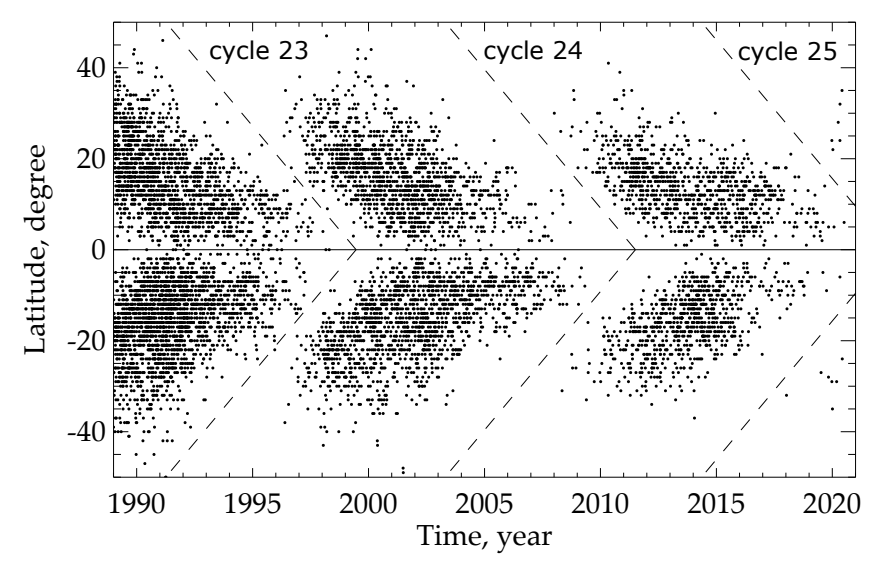

Figure 2. Time-latitude diagram of sunspot groups (by USAF/NOAA SRS data) with boundaries (dotted lines) between cycles plotted by McClintock, Norton, and Li (2014) technique.
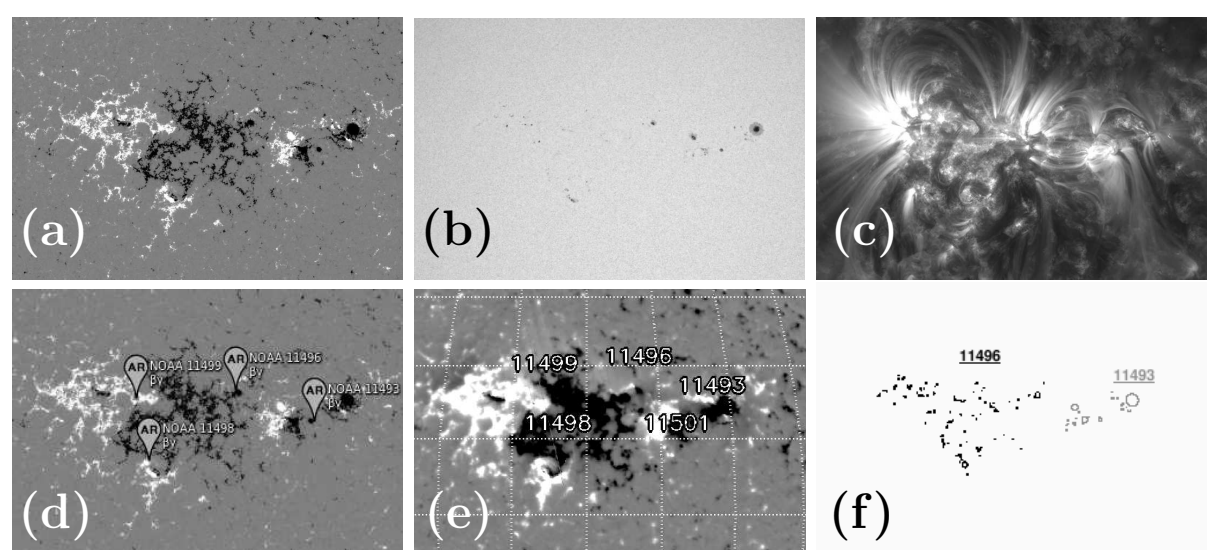

Figure 3. Composite activity complex observed on 06 June 2012 in the North hemisphere: HMI magnetogram (a), continuum (b), and AIA Fe IX/x $171 \AA$ line (c) images. Helioviewer (d), Solar Monitor (e), and DPD (f) interpretations of AR detection as an illustration of the difference in AR-separation algorithms. There are no anti-Hale regions in this block of ARs.

equal probability to several adjacent groups. Differences in segmentation of a magnetic complex by various tools is illustrated in Figure 3. We assumed that there are no anti-Hale sub-regions in this particular case.

iii) Phenomena in ARs in the late decaying stage when the following spots of an AR have vanished or weakened:

a) Presence of opposite polarity small sunspots/pores to the West of an old leading sunspot. To illustrate, an example of a large decaying AR is shown in Figure 4 Magnetic connections between a large leading spot of positive polarity and fragmented pores of negative polarity to the East are evident and steady (panels c, f). Such magnetic loops is the typical connection of the leading and the following parts of bipolar ARs. The absense of a magnetic connection between the large leading spot and a negative polarity pore to the South-West does not allow us to identify this couple of sunspots as a single AR with reverse polarity. 

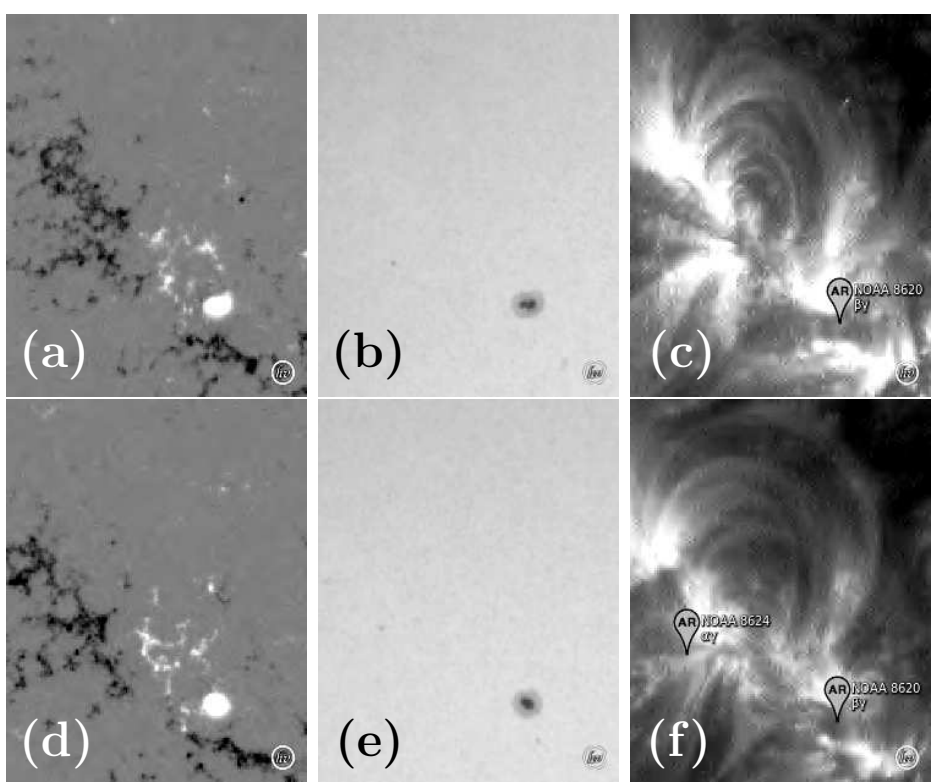

Figure 4. Sunspot group in the North hemisphere assigned by Helioviewer as a single AR 8620 on 06 July 1999: HMI magnetogram (a), continuum (b), and AIA Fe IX/X 171 A line (c) images; the same images of this sunspot group on 07 July (d, e, f): two separate ARs (NOAA 8620 and 8624) are assigned. A part of the mafgnetic structure assigned as NOAA 8620 on panel (f) is not a true anti-Hale AR because there is no magnetic connection between sunspots.

Note, however, that this couple of sunspots is assigned by Helioviewer as an individual AR NOAA 8620 on 07, 09 July 1999 and further; the following part of the initial sunspot group is extracted by Helioviewer as AR NOAA 8624. Such situation is typical for an AR with complex magnetic configuration (classified " $\gamma$ " in the Mount Wilson class).

Moving magnetic features might also cause emerging of pores on moat-cell boundaries (Ryutova, 2018). The combination of the old leading spot and opposite-polarity small spot/pore to the West of it may be misidentified as a separate sunspot group violating Hale's polarity law (Figure 5), especially by automatic algorithms.

b) Presence of opposite polarity pores all around the old sunspot. In cases when the flux (area) weighted center of scattered pores is located to the West of the old spot, the AR also might be erroneously identified as an anti-Hale region.

We did not include the above mentioned cases in the compiled catalog.

iv) Tilt instability of emerging ARs. The tilt is known to be unstable during several days after the emergence onset (see, e.g., van Driel-Gesztelyi and Green, 2015; Schunker et al., 2020) and may change the orientation importantly. One cannot determine the leading/following sunspot position and polarity unambiguously while the tilt is not stabilized. So, it is a challenge to establish a reverse polarity for the groups arising in the close proximity to the western limb, as well as for small short-lived ARs. We discarded ARs emerging near the western limb and observed only for one or two days. All short-lived ARs (having spots/pores 


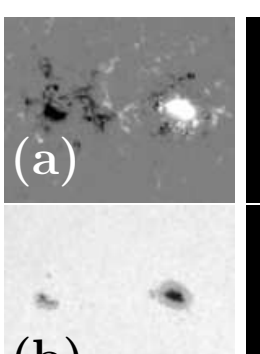

(b)
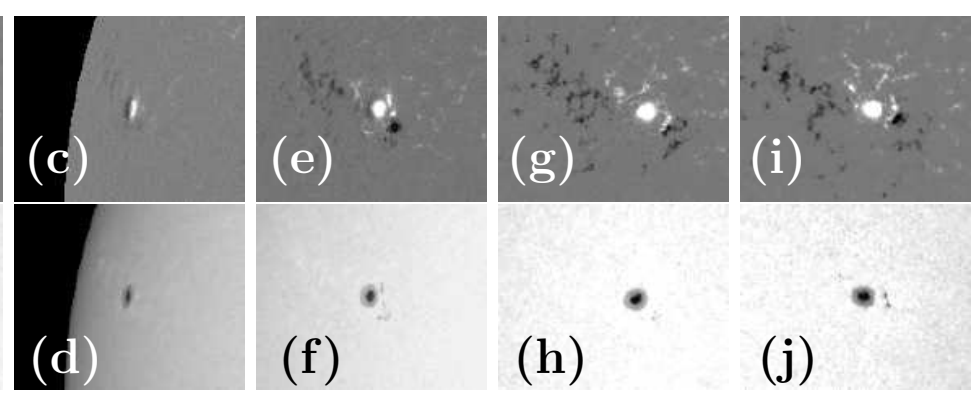

Figure 5. Recurrent AR NOAA 10634 (the North hemisphere) on 19 June 2004 (a,b) and its evolution as AR NOAA 10644 in the next rotation on 11 July 2004 (c,d), 13 (e,f), 15 $(\mathrm{g}, \mathrm{h}), 17$ (i,j) illustrated by MDI magnetograms (upper panel) and continuum images (lower panel). Spots/pores in the following polarity of AR NOAA 10644 are absent; the decay of the long-living leading sunspot is accompanied by the formation of small spots/pores on the moat-cell boundaries. The combination of this with the old leading sunspot might be misidentified as an anti-Hale AR.
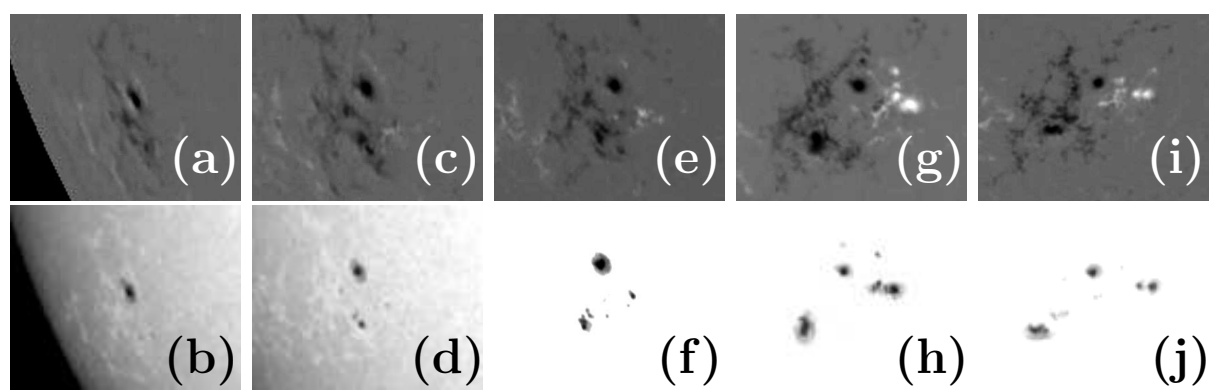

(b)
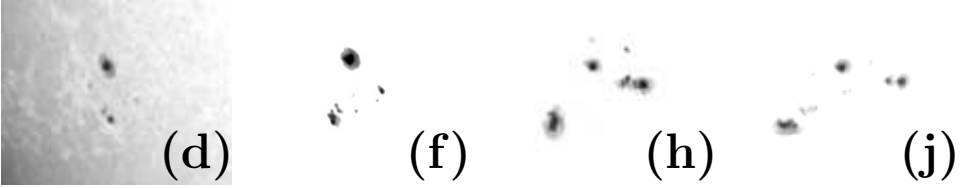

Figure 6. Evolution of AR NOAA 9264 (the South hemisphere) illustrated by MDI magnetograms (upper row) and continuum images (bottom row) on 11 December 2000 (a,b), 12 $(\mathrm{c}, \mathrm{d}), 13(\mathrm{e}, \mathrm{f}), 15(\mathrm{~g}, \mathrm{~h}), 17(\mathrm{i}, \mathrm{j})$. A new reverse-polarity bipole emerged near the old unipolar spot.

of both polarities for less than three days) and ARs with tilt uncertainties were indicated with special marks: "S" and "T", respectively (see Section 3.2).

v) Emergence of a new reverse-polarity bipole close to a pre-existing unipolar sunspot. Sometimes such new bipoles have an individual NOAA number, e.g. ARs NOAA 7062 close to 7056, and sometimes they do not. We extracted the new anti-Hale region from the whole activity complex (in the latter case, under the hosting NOAA number). In the catalog, they are marked by "X". A typical example is shown in Figure 6. This procedure was applied only for emerging antiHale regions that survived longer than three days. Sunspot areas for extracted ARs were calculated by using a technique described in Section 3.2.

vi) ARs changing their properties during the displacement across the disk. Some ARs, when passing across the disk, show anti-Hale properties for only a part of time. It may be due to emergence of a new magnetic flux in the vicinity. In other cases, a bipolar sunspot group with reverse-polarity remains after the decay of a multipolar AR. If the reverse-polarity property is observed longer than three days, we noted this region with a special mark "P". 

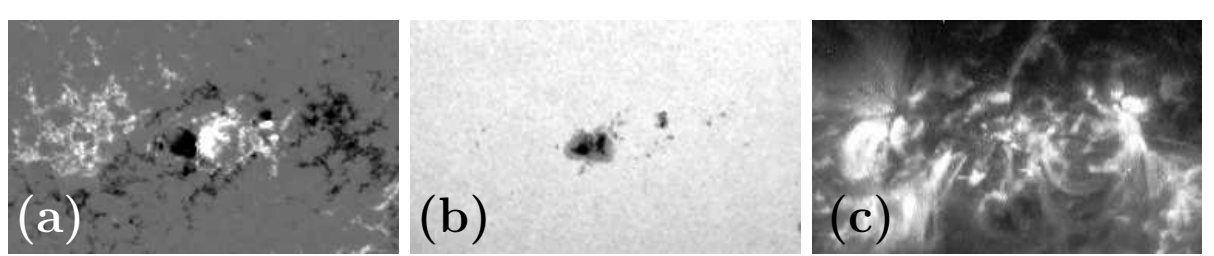

Figure 7. Complex AR NOAA 9591 including magnetic " $\delta$-configuration" sunspot observed on 28 August 2001 in the South hemisphere: MDI magnetogram (a), continuum (b), and AIA Fe IX/X 171 Å line (c) images. The " $\delta$-sunspot" of reverse polarity was extracted and considered in the compiled catalog.

vii) ARs with the main spots of both polarities near the Equator. McClintock, Norton, and Li (2014) argue that equatorial location of sunspot groups is related to a misalignment of the magnetic and heliographic Equators. They suppose that ARs such as NOAA 11987, for example, pertain to the magnetic system of the North hemisphere despite the fact that the leading spot is located a bit below the Equator. The authors explain such phenomena by the different degree of activity of the North and South hemispheres. Considering these reasons, we included into the catalog only those near-Equator ARs where the area-weighted center and the midpoint between the main spots of both polarities belong to the same hemisphere. They are marked with a letter "E".

viii) ARs with " $\delta$-configuration". This class of ARs was introduced for sunspot groups with closely spaced opposite-polarity umbrae located in the common penumbra and added to the initial Mount Wilson magnetic classification by Künzel (1965). A sample of AR NOAA 9591 including " $\delta$-sunspot" is presented as Figure [7. Individual " $\delta$-structures" with reverse polarity were included in our database with the mark " $D$ ". " $\delta$-spots" amidst the spread out background magnetic complex (see Figure 7) were extracted and considered only in the cases when such a " $\delta$-structure" was the strongest feature in the entire complex. In case the extracted " $\delta$-structure" displays the reverse polarity, we indicated it with the mark "W". There are only seven such events in the compiled catalog.

ix) Multipolar ARs. A sample of such AR (NOAA 10069) is shown in Figure8. a number of equivalent-by-size sunspots of both polarities are spread around without forming any single bipole. Sometimes the most western spots may have the wrong polarity according to the Hale's polarity law, see Figure 8. In spite of this attribute, we decided not to count multipolar ARs in out dataset. Examples of multipolar ARs are: NOAA 6562, 6624, 10501, 10786, and 12673.

$\mathrm{x})$ The polarity of sunspots may be reversed due to the projection effect. Of course, such cases were not counted.

\subsection{Shortcomings in the Existing Data Sources}

We also encountered issues related to data sources.

i) Flaws of the MWO data:

a) Lack of records (e.g. ARs NOAA 7926, 9858, and 10268) because of different reasons, including weather conditions (e.g. ARs NOAA 6255, 7430,7872, and $8963)$. 

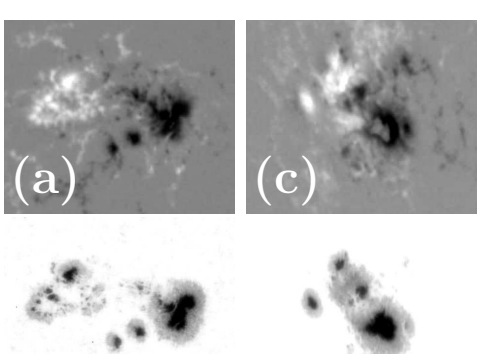

(b)
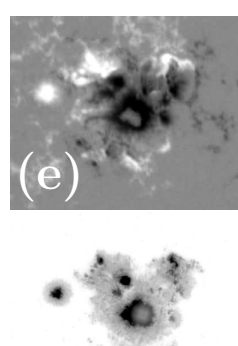

(f)
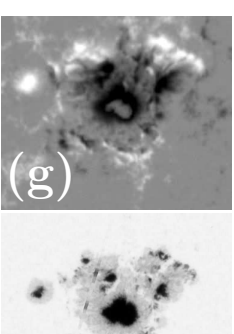

(h)
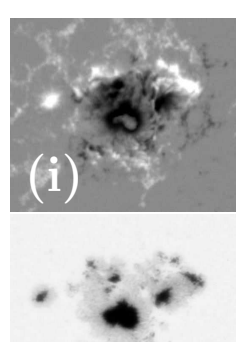

(j)

Figure 8. Recurrent AR NOAA 10036 (the South hemisphere) on 22 July 2002 (a,b) and its evolution as AR NOAA 10069 in the next rotation on 14 August 2002 (c,d), 16 (e,f), 17 $(\mathrm{g}, \mathrm{h}), 18(\mathrm{i}, \mathrm{j})$ illustrated by MDI magnetograms (upper row), DPD (Gyula Observing Station and Debrecen Heliophysical Observatory) continuum images (bottom row). The old leading sunspot is surrounded by opposite-polarity sunspots since 16 August; some of them are located at the western part of the AR. This one and similar multipolar ARs were not included in the catalog.
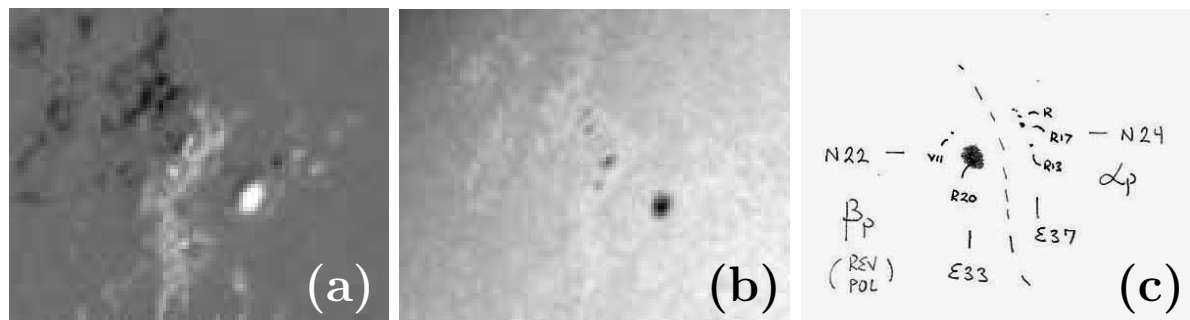

Figure 9. AR NOAA 9294 observed on 04 January 2001 in the North hemisphere: MDI magnetogram (a) and continuum image (b). (c) an erroneous identification of the group as a group of reverse polarity on the MWO drawing.

b) Wrong determination of the magnetic-field polarity (all sunspots/pores of a bipolar AR are marked as the same polarity features, e.g. ARs NOAA 8023, 9470, and 9699). Wang et al. (2015) also reports that such cases are multiple among ARs with the polar separation less than 2.5 degrees.

c) Erroneous usage or lack of the mark "REV. POL." (reversed polarity) on MWO drawings. A sample of an AR with proper polarity of the leading sunspot, but wrongly marked as "REV. POL." is presented in Figure 9 The magnetogram and the continuum image have the usual EW-orientation whereas the MWO drawing has the opposite orientation. The main spot R20 has a regular (for leading spots in Solar Cycle 23 in the North hemisphere) positive polarity, but the AR is marked as "REV. POL.". The opposite situation is observed in the MWO catalog for the anti-Hale ARs NOAA 5622, 7741, 7948, 8210, 9045, 9736, and 10638, which are not marked as reverse-polarity ARs.

d) Lack of AR NOAA number.

ii) Uncertainties in AR NOAA assignement in the DPD, the USAF/NOAA SRS databases, Solar Monitor, and Helioviewer:

a) After the decay of an AR, the same NOAA number was assigned to a new anti-Hale region that emerged at the same location (e.g. ARs NOAA 6241, 7324) or nearby (e.g. AR NOAA 7062); 
b) Differences in outlining of boundaries (areas) of ARs in the USAF/NOAA SRS and the DPD databases. For example, according to the DPD, the anti-Hale AR NOAA 12310 is a single active region. But according to the USAF/NOAA SRS and Solar Monitor, this complex is fragmented into three different ARs (NOAA 12310, 12312, and 12313). Another example: AR NOAA 6203 is classified as a single group on the DPD sketch, and as two separate ARs (including the anti-Hale bipolar group MWO number 26212) on the MWO drawing.

ARs with such ambiguities in various databases were indicated with mark "N".

iii) Errors in calculations of sunspot-group areas. For instance, in the USAF/NOAA SRS database on 13 July 1994 AR NOAA 7746 has an extremely high value of the group area, which triply exceeds that reported for previous and following days. At the same time, the DPD data show a smooth change of this value.

To this end, to provide a database of anti-Hale regions without the aforementioned shortcomings, we examined anew all ARs from 1989 to 2018.

\section{The Compiled Catalog}

\subsection{Anti-Hale Regions Selection Criteria}

When elaborating our criteria of identification of anti-Hale regions, we were guided by original ideas by Hale and Nicholson (1925). We also assumed that the main spots of different polarities are footpoints of emerged magnetic loops in accordance with classic magnetic-cycle models (Babcock, 1961; Parker, 1955). Following this, we formulated two basic criteria for identification of an AR as an anti-Hale region.

i) Dominating features of an AR form a bipole of reverse polarity with sunspots or pores of both polarities being present.

ii) Magnetic connections between the opposite polarities are steady: magnetic loops (arcades) are visible at least in one of the EUV images day by day (in the cases when EUV data are available).

The remaining four additional criteria are proposed for certain ambiguous types of ARs.

iii) For small, short-lived (less than three days) sunspot groups. The tilt of an $\mathrm{AR}$ has to be stable (not rotating, not perpendicular to the Equator). The rotation of the tilt more than 20 degrees during the interval of observation was considered as a non-stable tilt. The area of an AR must be greater than 4 $\mu \mathrm{Hem}$ and the corrected umbra area by the DPD must be non-zero (to exclude ambiguous pores: "transient" and "penumbrae without umbrae" features, see Lefevre and Clette 2014).

iv) For sunspot groups located on the Equator. Both the area-weighted center and the midpoint between the polarities of an AR have to be located in the same (the North or South) hemisphere.

v) For emerging bipolar groups appearing from a pre-existing AR. The extracted bipole has to exist at least three days. 
vi) For " $\delta$-structures" amidst the background magnetic complex. The extracted " $\delta$-structure" has to form the strongest feature in the magnetic complex and does not have steady magnetic connections with other magnetic centers of the complex.

We did not consider any unipolar sunspots. We also did not consider multipolar ARs.

\subsection{Anti-Hale Database and Special Marks}

All detected anti-Hale ARs are listed in the database (see the Electronic Supplementary Material). Several lines from the catalog beginning, as well as the two last lines of the catalog are shown as Table1. For each AR, the catalog includes three information blocks: i) the USAF/NOAA SRS data; ii) the DPD data; iii) our special marks. The first and second blocks each include the following parameters: the NOAA (or DPD) number; the date of the greatest corrected total area of sunspots in D M Y (day, month, year) format. For this date, we show: latitude (LAT), longitude (LON), the maximum value of the corrected total sunspots area (Area). Area values are provided in millionths of the solar hemisphere $(\mu \mathrm{Hem})$.

The most complex Mount Wilson magnetic class (Hale class) achieved by a given AR is also shown in the USAF/NOAA SRS data block. When the Mount Wilson magnetic class was not available, we assigned this class. For ARs that exhibit the anti-Hale behaviour only for a partial time during the evolution, we considered only the anti-Hale period to determine the Mount Wilson magnetic class.

The DPD block contains the tilt information for the ARs studied. Unfortunately, there is a lack of tilt data from 2014 to 2018 in the DPD database (except for some months in 2017). The sign "z" stands for missing data. The data on the recurrent ARs are provided in the catalog for each rotation separately.

We use the following special marks for particular types of ARs:

$\mathrm{C}$ - decaying (Collapsing) ARs;

$\mathrm{D}$ - individual " $\delta$-sunspots";

E - Equatorial ARs;

$\mathrm{N}$ - ARs with ambiguities in the NOAA numbers assignment and/or outlining of boundaries of sunspot groups in various databases;

$\mathrm{P}$ - an AR exhibits the anti-Hale property only $\mathbf{P a r t}$ time when passing across the disk;

$\mathrm{R}$ - ARs with Rotation of the sunspot-group axis;

$\mathrm{S}$ - small Short-lived ARs;

$\mathrm{T}-$ ARs with the Tilt close to $90^{\circ}$;

$\mathrm{V}$ - ARs which are not checked for the stability of magnetic connections (lack of EUV data);

W - " $\delta$-sunspots" With surrounding sunspots/pores;

$\mathrm{X}$ - eXtracted ARs.

The extracted ARs were counted with the hosting AR NOAA numbers. Area values in such cases were determined as follows. For the DPD data block, the 


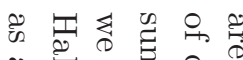

2 0 क 5

๑.

○

$\pm 曰 \curvearrowright \Omega$

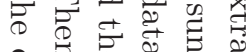

\& 3 है के

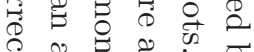

广 है :

+ G

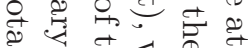

क 8 के

ह

㻤.

+ 导文豆

¿

$\circ \rightarrow 0$ 足

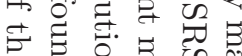

范

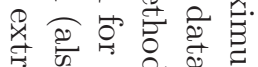

पै के ?

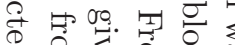

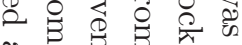

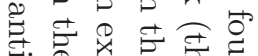

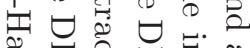

एँ

- 2 ¿

गे.

\begin{tabular}{|c|c|c|c|c|c|c|c|c|c|c|c|c|c|c|}
\hline & & & USAF & /NOAA & SRS & & & & DPD & & & & mal & rks \\
\hline No. & NOAA & $\mathrm{D} \quad \mathrm{M}$ & $1 \mathrm{Y}$ & $\begin{array}{l}\text { LAT, } \\
\text { [degr.] }\end{array}$ & $\begin{array}{l}\text { LON, } \\
\text { [degr.] }\end{array}$ & Area, $\mathrm{H}_{2}$ & $\begin{array}{l}\text { NOAA } \\
\text { /DPD }\end{array}$ & $\mathrm{D} \quad \mathrm{M} \mathrm{Y}$ & $\begin{array}{l}\text { LAT, } \\
\text { [degr.] }\end{array}$ & $\mathrm{LO}$ & $\begin{array}{l}\text { Area, } \\
\mu \mathrm{Hem}]\end{array}$ & $\begin{array}{c}\text { Tilt, } 1 \\
\text { [degr.] }\end{array}$ & & $\mathrm{C}$ \\
\hline 1 & 5409 & 2303 & 1989 & 18.0 & 14.0 & 990 BGD & 5409 & 22031989 & 16.79 & 12.46 & 1334 & -26.93 & $\mathrm{P}$ & V 1.00 \\
\hline 2 & 5414 & 2403 & 1989 & -18.0 & 49. & $150 \mathrm{~B}$ & 5414 & 21031989 & -18.01 & 20.65 & 238 & 26.55 & & V 1.00 \\
\hline 3 & 5441 & 1004 & 1989 & 36.0 & -15.0 & $330 \mathrm{BD}$ & 5441 & 08041989 & 35.03 & -29.55 & 506 & -24.66 & & V 1.00 \\
\hline 4 & 5455 & 1404 & 1989 & 19.0 & -58 & $20 \mathrm{~B}$ & 5455 & 15041989 & 24.93 & -31.74 & 6 & $\mathrm{~S}$ & & V 1.00 \\
\hline 5 & 5470 & 0505 & 1989 & 28.0 & -17.0 & 520 BGD & 5470 & 04051989 & 29.44 & -16.97 & 775 & 15.82 & & V 1.00 \\
\hline 6 & 5504 & 2405 & 1989 & 22.0 & -9. & $20 \mathrm{~B}$ & 5504 & 23051989 & 22.27 & -7.07 & 17 & $21.93 \mathrm{~S}$ & & V 1.00 \\
\hline 7 & 5548 & 1706 & 1989 & -22.0 & 58.0 & $10 \mathrm{~A}$ & 5548 & 16061989 & -21.55 & 57.96 & & $-47.77 \mathrm{~S}$ & $\mathrm{~T}$ & V 1.00 \\
\hline 8 & 5600 & 2207 & 1989 & -18.0 & -35.0 & $10 \mathrm{~B}$ & 5600 & 22071989 & -19.42 & -27.01 & 37 & zzzZz & $\mathrm{X}$ & V 0.52 \\
\hline 9 & 5622 & 0908 & 1989 & -26.0 & 44.0 & $280 \mathrm{~B}$ & 5622 & 08081989 & -26.07 & 42.26 & 444 & -13.28 & & V 1.00 \\
\hline 10 & 5648 & 1708 & 1989 & 7.0 & 14.0 & $10 \mathrm{~B}$ & 5648 & 17081989 & 6.80 & 29.83 & 9 & $-5.91 \mathrm{~S}$ & & NV 1.00 \\
\hline 11 & 5656 & 2308 & 1989 & 26.0 & -32.0 & $10 \mathrm{~B}$ & 5656 & 23081989 & 25.46 & -18.97 & 12 & $0.50 \mathrm{~S}$ & & V 1.00 \\
\hline 12 & 5703 & 2209 & 1989 & -23.0 & -45.0 & $60 \mathrm{~B}$ & 5703 & 21091989 & -22.14 & -44.66 & 72 & $19.27 \mathrm{C}$ & $\mathrm{P}$ & V 1.00 \\
\hline 13 & 5729 & 0610 & 1989 & -28.0 & -61.0 & $50 \mathrm{~B}$ & 5729 & 08101989 & -28.25 & -24.43 & 49 & -0.99 & & V 1.00 \\
\hline 14 & 5747 & 1910 & 1989 & -27.0 & -22.0 & $1160 \mathrm{BD}$ & 5747 & 19101989 & -26.72 & -12.04 & 1354 & -38.64 & DTRP & V 1.00 \\
\hline 274 & 12703 & 3103 & 2018 & -8.0 & -60.0 & & 703 & 30032018 & -9.17 & 1.68 & 24 & zzzz C & & 1.00 \\
\hline 275 & 12720 & 2608 & 2018 & 8.0 & 39.0 & $100 \mathrm{~B}$ & zzzzz & zz zz zzzz & zzzzzz & zzzzzz & zzzz & zzzzz & & 1.00 \\
\hline
\end{tabular}


to the corrected total sunspot area of the entire hosting group. This coefficient was applied to the area value of the hosting AR from the USAF/NOAA SRS database (at the same date) to calculate the anti-Hale region area. The auxiliary coefficient marked as "AC" is shown in the last column of the catalog.

Areas of ARs with shortcomings in areal data (see Section 2.3) were calculated by special procedure. For example, only the areas of relevant sunspots were counted for AR NOAA 12310 for the DPD data block.

\section{Estimations of the Relative Number of Anti-Hale Regions}

In total, we studied 8606 ARs of Solar Cycles 22, 23, and 24. 275 sunspot groups were identified as anti-Hale regions and included in the catalog. We found the anti-Hale percentage as $\approx 3.2$, and $\approx 3.0$ in the case of excluding short-lived ARs with unsteady tilt. This estimation is close to previous earliest results by Hale and Nicholson $(1925), \approx 2.4$, and by Richardson $(1948), \approx 3.1$.

However, as we show in the Introduction, the recent studies of anti-Hale regions provide a higher percentage of $4-8$. Possible reasons for this are discussed below.

We found that small short-lived groups (96 ARs) comprise about a one third of our set of anti-Hale regions. Exclusion of these ARs changes the anti-Hale percentage significantly (down to $\approx 2.0$ ). Since Richardson $(1948)$ ), it is well known that Hale's polarity-law violators happen more often for small $(<50 \mu \mathrm{Hem})$ sunspot groups. The frequency of this violation increases rather steeply with transition to regions of smaller size (Hagenaar, 2001; Tlatov, Vasil'eva, and Pevtsov, 2010; Stenflo and Kosovichev, 2012). Therefore, including smaller and smaller groups into a sample causes increases the anti-Hale region percentage.

The sensitivity of modern instruments (Scherrer et al., 1995, 2012) allows researchers to involve in the statistical studies weaker and weaker ARs. To estimate the magnetic-flux threshold for ARs selection in our study, we evaluated fluxes of several small short-lived ARs from the catalog. We found that the total unsigned magnetic-flux value of short-lived ARs having at least pores of both polarities (according to our criteria) is $\approx 10^{21} \mathrm{Mx}$. This allows us to consider this flux value as an indirect estimate of the threshold for our selection. We presume that counting of ARs with smaller magnetic fluxes is one of the main reasons for the enhanced percentage of anti-Hale groups in recent studies.

Another reason for the discrepancy might be the numerous ambiguities in antiHale region identification mentioned in Section 2.2. For example, the detection of anti-Hale ARs in activity complexes is very challenging (Section 2.2, item 2). According to data by Yazev (2011, 2015), there are 321 activity complexes in Solar Cycles 22-24, which corresponds $900-1100$ ARs (9-12\% from all ARs). Using average evaluation of anti-Hale ARs percentage $\approx 4.5$ (see the Introduction), we roughly estimate a possible number of anti-Hale regions in activity complexes for three mentioned cycles as $40-50$ ARs (up to $0.5 \%$ from all ARs). This is an upper limit of anti-Hale ARs number which might be missed in our catalog. 
We also compared the anti-Hale regions list for 1989-2004 that was used by Sokoloff, Khlystova, and Abramenko (2015) (the 2015 database) with the cotemporal fragment of the compiled catalog. We found that some changes occured in the list of anti-Hale regions; the number of ARs in both databases remains close. The changes turned out to be evenly distributed along the cycle, so that the main inference made by Sokoloff, Khlystova, and Abramenko (2015) about the cycle variations of the relative number of anti-Hale groups did not change.

\section{Conclusions}

To identify ARs violating the Hale's polarity law, we visually examined sunspot groups from 1 January 1989 to 31 December 2018. This period encompasses the end of the Solar Cycle 22 and the two subsequent cycles including two solar minima, which is important for understanding the coupling between local and global magnetic fields in terms of solar dynamo theory. We used the SOHO/MDI and the SDO/HMI data, as well as the DPD sketches and the MWO catalog and drawings. Detailed comparison of data from different source allowed us to detect a number of ambiguous cases in anti-Hale sunspot groups identification. We analysed the revealed errors and inaccuracies to elaborate the criteria for identification of sunspot groups as anti-Hale ARs. We can conclude the following.

i) The main reasons for possible errors in anti-Hale region identification are:

a) incorrect determination of AR boundaries;

b) wrongly distributing of ARs between consecutive cycles during solar minima;

c) presence of small sunspots/pores with an opposite (to a proper leading polarity by the Hale's law) polarity to the West from the old leading spots in decaying ARs;

d) emergence of a new magnetic flux within decaying ARs;

e) the wrong polarity of the most western spots in multipolar ARs;

f) location of an AR on the Equator;

g) appearance of the wrong polarity of sunspots located near the limb the projection effect).

ii) Two basic and four additional criteria for identifying bipolar sunspot groups as anti-Hale regions are formulated. Our basic criteria are based on the classical ideas of Hale and Nicholson (1925) and Babcock (1961). Namely, we assume that for an AR to be classified as an anti-Hale region, its dominating features have to form a bipole of reverse polarity with sunspots/pores of both polarities being present. Magnetic connection between the opposite polarities has to be observed.

iii) The catalog of anti-Hale regions for the period of our study is compiled. For each anti-Hale region, the catalog contains: NOAA (or DPD) number; date, coordinates, and corrected total sunspot area at the evolutionary maximum as determined from the DPD and the USAF/NOAA SRS data. The catalog also contains the tilt and Mount Wilson magnetic class. In total, the catalog contains 275 ARs, including 96 small short-lived sunspot groups. 
iv) The percentage of sunspot groups violating the Hale's polarity law (meeting the formulated criteria) is $\approx 3.0$ from all studied ARs. This is close to the earliest estimations by the authors who had examined each AR individually: $\approx 2.4$ by Hale and Nicholson (1925) and $\approx 3.1$ by Richardson (1948). Later researchers reported a slightly higher percentage. The enchancement of the percentage might be related to increasing sensitivity of instruments and, therefore, taking into account smaller and smaller bipoles. Another reason for the discrepancy in anti-Hale percentage is the ambiguities in the anti-Hale regions identification mentioned in Section 2.2.

The catalog of anti-Hale regions can be used in studying the phenomena related to the cyclic solar activity. The catalog is available as the Electronic Supplementary Material. The catalog is also available at the CrAO website sun.crao.ru/databases/catalog-anti-hale.

Acknowledgments The authors are thankful to Drs. L.V. Ermakova and A.S. Kutsenko for consulting on complex, ambigous ARs. Efforts of A.V.Zhukova on the catalog compilation were supported by the RSF (Project 18-12-00131). A.I.Khlystova thanks the support of the RFBR's grants 18-02-00085 and 19-52-45002. The work of A.I.Khlystova was supported by the basic financial program of the FSR II.16. D.D.Sokoloff is grateful for the RFBR support under the grant 18-02-00085. V.I.Abramenko would like to acknowledge partial support of the MSHE of RF (Research 0831-2019-0006).

\section{Disclosure of Potential Conflicts of Interest}

The authors declare that they have no conflicts of interest.

\section{References}

Abramenko, V.I., Zhukova, A.V., Kutsenko, A.S.: 2018, Contributions from Different-Type Active Regions Into the Total Solar Unsigned Magnetic Flux. Geomag. Aeron. 58, 1159. DOI ADS

Babcock, H.W.: 1961, The Topology of the Sun's Magnetic Field and the 22-YEAR Cycle. Astrophys. J. 133, 572. DOI ADS

Baranyi, T., Győri, L., Ludmány, A.: 2016, On-line Tools for Solar Data Compiled at the Debrecen Observatory and Their Extensions with the Greenwich Sunspot Data. Solar Phys. 291, 3081. DOI ADS

Blackman, E.G., Field, G.B.: 2002, New Dynamical Mean-Field Dynamo Theory and Closure Approach. Phys. Rev. Lett. 89, 265007. DOI ADS

Brandenburg, A., Subramanian, K.: 2005, Astrophysical magnetic fields and nonlinear dynamo theory. Phys. Rep. 417, 1. DOI ADS

Brandenburg, A., Sokoloff, D., Subramanian, K.: 2012, Current Status of Turbulent Dynamo Theory. From Large-Scale to Small-Scale Dynamos. Space Sci. Rev. 169, 123. DOI. ADS.

Hagenaar, H.J.: 2001, Ephemeral Regions on a Sequence of Full-Disk Michelson Doppler Imager Magnetograms. Astrophys. J. 555, 448. DOI. ADS.

Hale, G.E., Nicholson, S.B.: 1925, The Law of Sun-Spot Polarity. Astrophys. J. 62, 270. DOI ADS

Hale, G.E., Ellerman, F., Nicholson, S.B., Joy, A.H.: 1919, The Magnetic Polarity of Sun-Spots. Astrophys. J. 49, 153. DOI ADS

Hazra, G., Choudhuri, A.R., Miesch, M.S.: 2017, A Theoretical Study of the Build-up of the Sun's Polar Magnetic Field by using a 3D Kinematic Dynamo Model. Astrophys. J. 835, 39. DOI ADS 
Howard, R.F.: 1989, The Magnetic Fields of Active Regions - Part One. Solar Phys. 123, 271. DOI ADS

Jiang, J., Cameron, R.H., Schüssler, M.: 2015, The Cause of the Weak Solar Cycle 24. Astrophys. J. Lett. 808, L28. DOI ADS

Kazantsev, A.P.: 1968, Enhancement of a Magnetic Field by a Conducting Fluid. Sov. J. Exp. Theor. Phys. 26, 1031. ADS

Khlystova, A.I., Sokoloff, D.D.: 2009, Toroidal magnetic field of the Sun from data on Halerule-violating sunspot groups. Astron. Rep. 53, 281. DOI ADS

Kraichnan, R.H., Nagarajan, S.: 1967, Growth of Turbulent Magnetic Fields. Phys. Fl. 10, 859. DOI ADS

Krause, F., Rädler, K.-H.: 1980, Mean-field magnetohydrodynamics and dynamo theory. ADS

Künzel, H.: 1965, Zur Klassifikation von Sonnenfleckengruppen. Astron. Nachr. 288, 177. ADS

Lefevre, L., Clette, F.: 2014, Survey and Merging of Sunspot Catalogs. Solar Phys. 289, 545. DOI ADS

Leighton, R.B.: 1964, Transport of Magnetic Fields on the Sun. Astrophys. J. 140, 1547. DOI ADS

Lemen, J.R., Title, A.M., Akin, D.J., Boerner, P.F., Chou, C., Drake, J.F., Duncan, D.W., Edwards, C.G., Friedlaender, F.M., Heyman, G.F., Hurlburt, N.E., Katz, N.L., Kushner, G.D., Levay, M., Lindgren, R.W., Mathur, D.P., McFeaters, E.L., Mitchell, S., Rehse, R.A., Schrijver, C.J., Springer, L.A., Stern, R.A., Tarbell, T.D., Wuelser, J.-P., Wolfson, C.J., Yanari, C., Bookbinder, J.A., Cheimets, P.N., Caldwell, D., Deluca, E.E., Gates, R., Golub, L., Park, S., Podgorski, W.A., Bush, R.I., Scherrer, P.H., Gummin, M.A., Smith, P., Auker, G., Jerram, P., Pool, P., Soufli, R., Windt, D.L., Beardsley, S., Clapp, M., Lang, J., Waltham, N.: 2012, The Atmospheric Imaging Assembly (AIA) on the Solar Dynamics Observatory (SDO). Solar Phys. 275, 17. DOI ADS

Li, J.: 2018, A Systematic Study of Hale and Anti-Hale Sunspot Physical Parameters. Astrophys. J. 867, 89. DOI ADS

Li, J., Ulrich, R.K.: 2012, Long-term Measurements of Sunspot Magnetic Tilt Angles. Astrophys. J. 758, 115. DOI ADS

López Fuentes, M.C., Demoulin, P., Mandrini, C.H., van Driel-Gesztelyi, L.: 2000, The Counterkink Rotation of a Non-Hale Active Region. Astrophys. J. 544, 540. DOI ADS

López Fuentes, M.C., Démoulin, P., Mandrini, C.H., Pevtsov, A.A., van Driel-Gesztelyi, L.: 2003, Magnetic twist and writhe of active regions. On the origin of deformed flux tubes. Astron. Astrophys. 397, 305. DOI ADS

McClintock, B.H., Norton, A.A., Li, J.: 2014, Re-examining Sunspot Tilt Angle to Include Anti-Hale Statistics. Astrophys. J. 797, 130. DOI ADS

Moffatt, H.K.: 1978, Magnetic Field Generation in Electrically Conducting Fluids, Cambridge Univ. Press, Cambridge, UK. ADS

Mordvinov, A.V., Kitchatinov, L.L.: 2019, Evolution of the Sun's Polar Fields and the Poleward Transport of Remnant Magnetic Flux. Solar Phys. 294, 21. DOI ADS

Moses, D., Clette, F., Delaboudinière, J.-P., Artzner, G.E., Bougnet, M., Brunaud, J., Carabetian, C., Gabriel, A.H., Hochedez, J.F., Millier, F., Song, X.Y., Au, B., Dere, K.P., Howard, R.A., Kreplin, R., Michels, D.J., Defise, J.M., Jamar, C., Rochus, P., Chauvineau, J.P., Marioge, J.P., Catura, R.C., Lemen, J.R., Shing, L., Stern, R.A., Gurman, J.B., Neupert, W.M., Newmark, J., Thompson, B., Maucherat, A., Portier-Fozzani, F., Berghmans, D., Cugnon, P., van Dessel, E.L., Gabryl, J.R.: 1997, EIT Observations of the Extreme Ultraviolet Sun. Solar Phys. 175, 571. DOI ADS

Parker, E.N.: 1955, Hydromagnetic Dynamo Models. Astrophys. J. 122, 293. DOI ADS.

Rädler, K.-H., Kleeorin, N., Rogachevskii, I.: 2003, The Mean Electromotive Force for MHD Turbulence: The Case of a Weak Mean Magnetic Field and Slow Rotation. Geophys. Astrophys. Fluid Dyn. 97, 249. DOI ADS

Richardson, R.S.: 1948, Sunspot Groups of Irregular Magnetic Polarity. Astrophys. J. 107, 78. DOI ADS

Ryutova, M.: 2018, Physics of Magnetic Flux Tubes. Astrophys. Space Sci. Lib. 455. DOI ADS

Scherrer, P.H., Bogart, R.S., Bush, R.I., Hoeksema, J.T., Kosovichev, A.G., Schou, J., Rosenberg, W., Springer, L., Tarbell, T.D., Title, A., Wolfson, C.J., Zayer, I., MDI Engineering Team: 1995, The Solar Oscillations Investigation - Michelson Doppler Imager. Solar Phys. 162, 129. DOI ADS 
Scherrer, P.H., Schou, J., Bush, R.I., Kosovichev, A.G., Bogart, R.S., Hoeksema, J.T., Liu, Y., Duvall, T.L., Zhao, J., Title, A.M., Schrijver, C.J., Tarbell, T.D., Tomczyk, S.: 2012, The Helioseismic and Magnetic Imager (HMI) Investigation for the Solar Dynamics Observatory (SDO). Solar Phys. 275, 207. DOI ADS.

Schunker, H., Baumgartner, C., Birch, A.C., Cameron, R.H., Braun, D.C., Gizon, L.: 2020, Average motion of emerging solar active region polarities. II. Joy's law. Astron. Astrophys. 640, A116. DOI ADS

Smith, S.F., Howard, R.: 1968, Magnetic Classification of Active Regions. In: Kiepenheuer, K.O. (ed.) Structure and Development of Solar Active Regions, IAU Symp. 35, 33. ADS.

Sokoloff, D., Khlystova, A.I.: 2010, The solar dynamo in the light of the distribution of various sunspot magnetic classes over butterfly diagram. Astron. Nachr. 331, 82. DOI ADS

Sokoloff, D., Khlystova, A., Abramenko, V.: 2015, Solar small-scale dynamo and polarity of sunspot groups. Mon. Not. Roy. Astron. Soc. 451, 1522. DOI ADS

Stenflo, J.O., Kosovichev, A.G.: 2012, Bipolar Magnetic Regions on the Sun: Global Analysis of the SOHO/MDI Data Set. Astrophys. J. 745, 129. DOI ADS.

Sur, S., Brandenburg, A., Subramanian, K.: 2008, Kinematic $\alpha$-effect in isotropic turbulence simulations. Mon. Not. Roy. Astron. Soc. 385, L15. DOI ADS

Tlatov, A.G., Vasil'eva, V.V., Pevtsov, A.A.: 2010, Distribution of Magnetic Bipoles on the Sun over Three Solar Cycles. Astrophys. J. 717, 357. DOI ADS

van Driel-Gesztelyi, L., Green, L.M.: 2015, Evolution of Active Regions. Liv. Rev. Solar Phys. 12, 1. DOI ADS.

Vitinsky, Y.I.: 1986, On Sunspot Groups with Irregular Magnetic Polarities. Byull. Solnechnye Dannye Akad. Nauk SSSR 9, 86. ADS.

Wang, Y.-M., Sheeley, J. N. R.: 1989, Average Properties of Bipolar Magnetic Regions during Sunspot CYCLE-21. Solar Phys. 124, 81. DOI ADS

Wang, Y.-M., Colaninno, R.C., Baranyi, T., Li, J.: 2015, Active-region Tilt Angles: Magnetic versus White-light Determinations of Joy's Law. Astrophys. J. 798, 50. DOI. ADS.

Yazev, S.A.: 2011, Activity complexes on the sun during the 23rd solar cycle. Geomag. Aeron. 51, 879. DOI. ADS.

Yazev, S.A.: 2015, Activity complexes on the sun in solar cycle 24. Astron. Rep. 59, 228. DOI ADS

Zeldovich, Y.B., Ruzmaikin, A.A., Sokoloff, D.D.: 1990, The Almighty Chance, World Scientific, Singapore. DOI ADS.

Zhukova, A.: 2018, A catalog of active regions of the 24th solar cycle. Izv. Krym. Astrofiz. Obs. 114, 74 